\title{
Cutaneous chemotherapy-induced radiation recall reaction
}

\author{
Michael J. McKay ${ }^{1}$, Jeremy McKay ${ }^{2}$, Monica Dumbrava ${ }^{1}$, and Thomas McKay ${ }^{3}$ \\ ${ }^{1}$ Northern Cancer Service, Tasmania \\ ${ }^{2}$ Deakin University \\ ${ }^{3}$ Monash University
}

February 6, 2021

\begin{abstract}
An 80 year old man with metastatic castrate-resistant prostate cancer was treated with multiple systemic agents but after carboplatin/paclitaxel, developed a morbilliform rash conforming precisely to a skin site of previous palliative radiotherapy, a so-called radiation recall reaction.
\end{abstract}

An 80 year old man with metastatic castrate resistant prostate cancer had previously had external beam radiotherapy to the prostate (76Gy, 38 fractions) in 2018, with the development of metastatic bone disease in mid 2019. He was commenced on androgen deprivation therapy with Zoladex/Cosudex, but progressed in bones and was started on Enzalutamide. In March 2020 he received palliative radiotherapy to the right scapula (20Gy, 5 fractions, Figure 1a). He was commenced sequentially on Docetaxel and Cabazitaxel and with further progressive disease, Carboplatin/Paclitaxel was instituted in December 2020: this resulted in the development of a pruritic, morbilliform cutaneous rash, conforming to the site of previous scapular radiotherapy (Figure 1b). Could the rash be associated with previous radiotherapy? It is very likely that he suffered a chemotherapy-induced radiation recall reaction. This well-described but poorly understood phenomenon most commonly affects skin but can affect other organs (1). It has been described after multiple chemotherapeutic agents (including carboplatin and taxane therapy as used here (2).

\section{References}

1. Azria, D et al. Radiation recall: a well recognised but neglected phenomenon. Cancer Treat Reviews (2005) 31, 555-570. 2. Chao, H-H et al. Radiation recall myositis after carboplatin/docetaxel chemotherapy (2020) 10, e50-e52. 


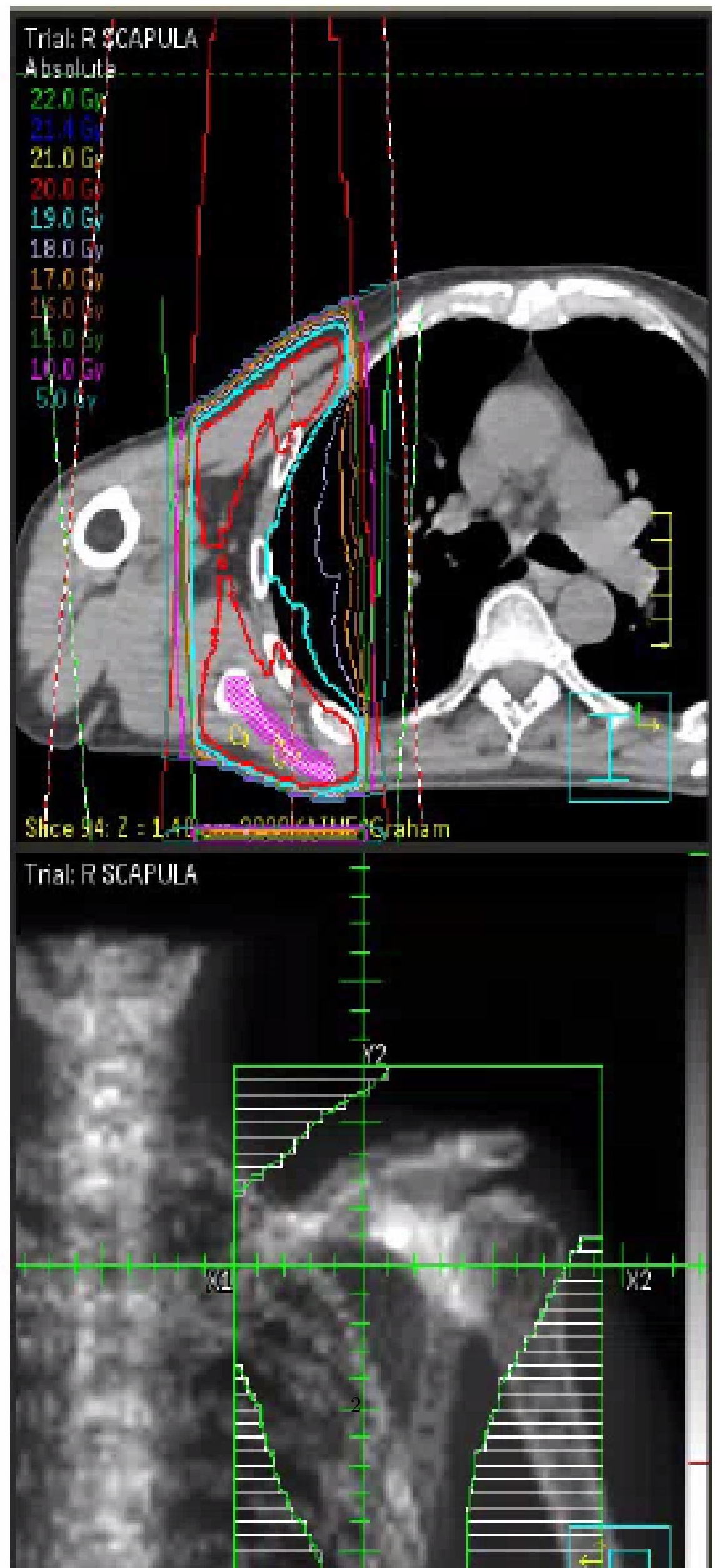




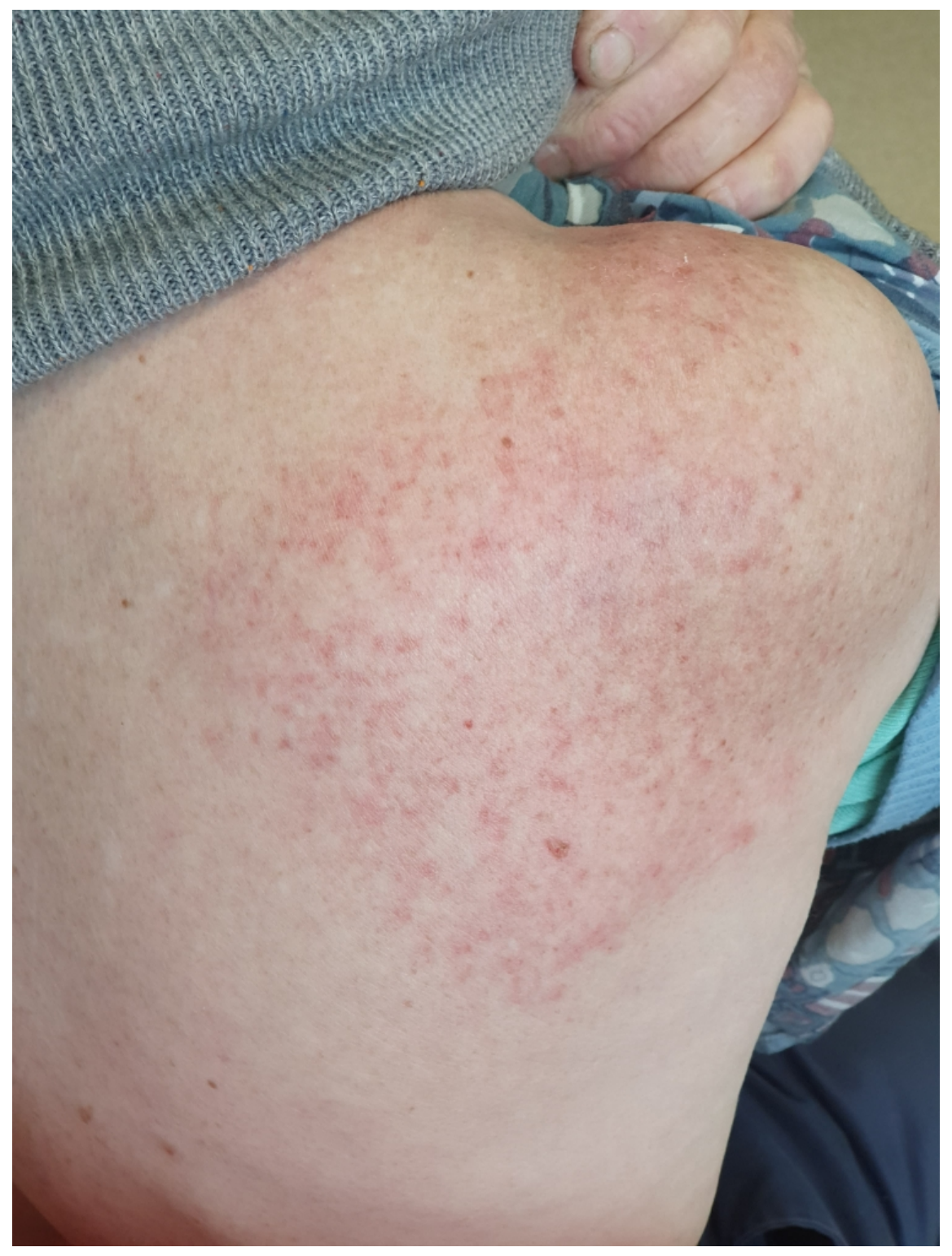

